\title{
Virologic Response by Baseline Viral Load With Dolutegravir Plus Lamivudine vs Dolutegravir Plus Tenofovir Disoproxil Fumarate/Emtricitabine: Pooled Analysis
}

\author{
Joseph Eron, MD, ${ }^{a}$ Chien-Ching Hung, MD, ${ }^{b}$ Jean-Guy Baril, MD, ${ }^{c}$ Jihad Slim, MD, ${ }^{d}$ Vicenç Falcó, MD, ${ }^{c}$ \\ Johannes Bogner, MD, ${ }^{f}$ Franco Maggiolo, MD, ${ }^{g}$ Anthony Mills, MD, ${ }^{h}$ Jörg Sievers, DPhil, ${ }^{i}$ \\ Choy Y. Man, BSc, ${ }^{j}$ Rimgaile Urbaityte, MStat, ${ }^{k}$ Mark Underwood, PhD, ${ }^{j}$ Allan R. Tenorio, MD, ${ }^{j}$ \\ Keith A. Pappa, PharmD, ${ }^{j}$ Brian Wynne, MD, Justin Koteff, PharmD, ${ }^{j}$ Martin Gartland, PhD, \\ Kimberly Y. Smith, $M D^{j}$, and Michael Aboud, $M D^{i}$
}

\begin{abstract}
Background: To investigate antiviral potency of the 2-drug regimen (2DR) dolutegravir plus lamivudine vs the 3-drug regimen (3DR) dolutegravir plus tenofovir disoproxil fumarate/emtricitabine, we performed a post-hoc analysis assessing antiviral response rates in the phase III GEMINI-1 and GEMINI-2 studies by baseline viral load (VL).
\end{abstract}

Setting: One hundred ninety-two centers in 21 countries.

Methods: Treatment-naive HIV-1-infected participants with screening VL $\leq 500,000$ copies $/ \mathrm{mL}$ were randomized 1:1 to oncedaily dolutegravir plus lamivudine or dolutegravir plus tenofovir disoproxil fumarate/emtricitabine. Median change from baseline was determined for $\log _{10}$-transformed VL in the overall study population and the subpopulation with baseline VL $>100,000$ copies $/ \mathrm{mL}$. Proportion of participants achieving plasma VL $<50$ copies $/ \mathrm{mL}$
(Snapshot algorithm) or $<40$ copies/mL (Abbott RealTime HIV-1 assay) and target not detected was assessed through week 48 by baseline VL. Time to viral suppression was determined (nonparametric Kaplan-Meier method).

Results: For 293 participants with baseline VL $>100,000$ copies $/ \mathrm{mL}$, median change from baseline at week 4 was -3.38 and $-3.40 \log _{10}$ copies/mL in the $2 \mathrm{DR}$ and $3 \mathrm{DR}$ groups, respectively; reduction was sustained throughout 48 weeks. Time to VL $<50$ copies $/ \mathrm{mL}$ was longer in participants with baseline VL $>100,000$ copies/mL than the overall study population (57 [week 8] vs 29 days [week 4]) and similar between the 2DR and 3DR groups. Proportion of participants with VL $<50$ or $<40$ copies $/ \mathrm{mL}$ and target not detected was similar between groups, irrespective of baseline VL, at all tested visits throughout 48 weeks.

Received for publication July 30, 2019; accepted October 23, 2019.

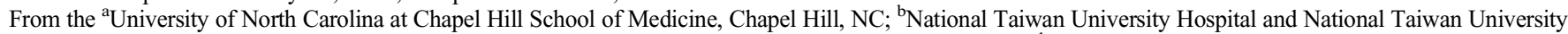
College of Medicine, Taipei, Taiwan; 'Clinique Médicale du Quartier Latin, Montréal, Québec, Canada; ${ }^{\text {'Saint Michael's Medical Center, Newark, NJ; }{ }^{~} H o s p i t a l ~}$

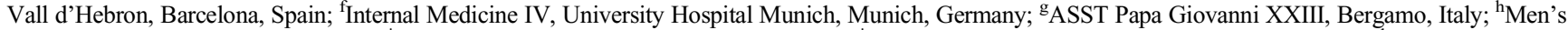
Health Foundation, Los Angeles CA; ${ }^{\mathrm{i}}$ ViiV Healthcare, Brentford, United Kingdom; ${ }^{\mathrm{j}}$ ViiV Healthcare, Research Triangle Park, NC; and ${ }^{\mathrm{k}}$ GlaxoSmithKline, London, United Kingdom.

Supported by ViiV Healthcare. Editorial assistance was provided under the direction of the authors by Leila Strickland, PhD, CMPP, and Jennifer Rossi, MA, ELS, MedThink SciCom, and was funded by ViiV Healthcare.

J.E. has received honoraria for consulting for Merck, ViiV Healthcare, Janssen, and Gilead Sciences. The University of North Carolina receives research support for clinical trials for which Dr Eron is an investigator. C.-C.H. has received honoraria for speaking at educational events or consulting for AbbVie, BristolMyers Squibb, Gilead Sciences, Janssen, and ViiV Healthcare and has received research funding from Bristol-Myers Squibb, Janssen, Gilead Sciences, Merck, and ViiV Healthcare. J.-G.B has received honoraria for consulting for ViiV Healthcare, Merck, and Gilead Sciences and for participation as a speaker at conferences from Merck and Gilead Sciences. His institution, Clinique Médicale du Quartier Latin, has received research grants from GlaxoSmithKline, Merck, and Gilead Sciences. V.F. has received honoraria for speaking at educational events from Janssen, Merck, and ViiV Healthcare and has received research funding from Gilead Sciences, Merck, and ViiV Healthcare. J.B. has received personal fees for participation as a speaker from AbbVie, Gilead Sciences, Janssen, Merck, Hexal, and ViiV Healthcare and for participation in advisory boards from Gilead Sciences, Janssen, Merck, and ViiV Healthcare. F.M. has received research funding from ViiV Healthcare, Gilead Sciences, Merck, Janssen, and AbbVie and has received personal fees for participation in advisory boards from ViiV Healthcare, Gilead Sciences, and Merck. A.M. has received honoraria for consulting for Merck, ViiV Healthcare, Janssen, and Gilead Sciences. The Men's Health Foundation receives research support for clinical trials for which Dr Mills is an investigator. J.S., C.Y.M., M.U., A.R.T., K.A.P., B.W., J.K., M.G., K.Y S., and M.A. are employees of ViiV Healthcare and own stock in GlaxoSmithKline. R.U. is an employee of GlaxoSmithKline and owns stock in GlaxoSmithKline. The remaining author has no conflicts to disclose.

J.S., A.R.T., K.A.P., B.W., M.G., K.Y.S., and M.A. contributed to the conception of the study. J.S., C.Y.M., R.U., M.U., A.R.T., K.A.P., B.W., M.G., K.Y.S., and M.A. contributed to the design of the study. J.S., C.Y.M., R.U., M.U., A.R.T., and B.W. contributed to the acquisition of data. J.S., C.Y.M., R.U., M.U., A.R.T., K.A.P., B.W., J.K., M.G., K.Y.S., and M.A. contributed to the analysis of data. All authors contributed to the interpretation of the data, drafting the manuscript, and critically revising the manuscript for important intellectual content, and all authors approve the manuscript for publication.

Anonymized individual participant data and study documents can be requested for further research from www.clinicalstudydatarequest.com.

Correspondence to: Joseph Eron, MD, University of North Carolina at Chapel Hill School of Medicine, CB\# 7030 , Bioinformatics Building, 130 Mason Farm Road, 2nd Floor, Chapel Hill, NC 27599-7030 (e-mail: joseph_eron@med.unc.edu).

Copyright (C) 2020 The Author(s). Published by Wolters Kluwer Health, Inc. This is an open-access article distributed under the terms of the Creative Commons Attribution-Non Commercial-No Derivatives License 4.0 (CCBY-NC-ND), where it is permissible to download and share the work provided it is properly cited. The work cannot be changed in any way or used commercially without permission from the journal. 
Conclusion: Dolutegravir plus lamivudine demonstrates high antiviral potency in treatment-naive HIV-1-infected individuals across baseline VL strata.

Key Words: HIV-1 infection, antiretroviral therapy, virologic response, 2-drug regimen, treatment-naive, viral load

(J Acquir Immune Defic Syndr 2020;84:60-65)

\section{INTRODUCTION}

The introduction of combination antiretroviral therapy (ART) has led to substantial increases in life expectancy among people living with HIV (PLHIV). ${ }^{1}$ As mortality rates among PLHIV decline and life expectancy approaches that of the general population, ${ }^{1}$ concerns about adverse effects associated with long-term ART use have prompted interest in reducing the number of drugs in antiretroviral regimens to minimize cumulative drug exposure. ${ }^{2}$

The phase III GEMINI-1 and GEMINI-2 studies demonstrated noninferior virologic efficacy for the 2-drug regimen $(2 \mathrm{DR})$ dolutegravir plus lamivudine compared with the 3-drug regimen (3DR) dolutegravir plus tenofovir disoproxil fumarate/emtricitabine fixed-dose combination at week 48 for treatment-naive participants with screening HIV-1 viral load (VL) $\leq 500,000$ copies $/ \mathrm{mL}^{3}$ In these studies, 716 and 717 participants received the $2 \mathrm{DR}$ and 3DR, respectively; only 6 and 4 participants met protocoldefined virologic withdrawal criteria through week 48, and no treatment-emergent resistance to integrase strand transfer inhibitors or nucleoside reverse transcriptase inhibitors (NRTIs) occurred. ${ }^{3}$

The efficacy of an antiretroviral regimen in patients with high VL is an important indicator of regimen potency. ${ }^{4}$ Ritonavir-boosted darunavir plus raltegravir is a 2DR that failed to demonstrate noninferiority to a standard-of-care 3DR in participants with baseline VL $>100,000$ copies/mL despite demonstrating noninferiority in the overall study population. ${ }^{5}$ To evaluate the potency of the 2DR dolutegravir plus lamivudine, virologic response in participants by baseline VL was analyzed post-hoc using pooled data from the GEMINI studies.

\section{METHODS}

The GEMINI studies were identically designed, doubleblind, randomized, phase III trials conducted at 192 centers in 21 countries in participants aged $\geq 18$ years with HIV-1 infection who were ART-naive. The GEMINI studies initially included participants with screening VL 1000 to 100,000 copies/mL; however, as supported by the protocol, the upper limit was increased to 500,000 copies/mL after an independent review of data from other trials investigating dolutegravir plus lamivudine (methodology described previously). ${ }^{3}$ In some participants with screening VL $\leq 500,000$ copies $/ \mathrm{mL}$, VL exceeded $>500,000$ copies $/ \mathrm{mL}$ at baseline. Those with hepatitis B or pre-existing major mutations conferring resistance to NRTIs, nonnucleoside reverse transcriptase inhibitors, or protease inhibitors were excluded.
Written informed consent was obtained from each participant before study entry, and study protocols were approved by appropriate ethics committees and institutional review boards in accordance with the Declaration of Helsinki and the International Conference on Harmonization Guideline for Good Clinical Practice.

Participants were randomized $1: 1$ to a once-daily oral regimen of either dolutegravir $(50 \mathrm{mg})$ plus lamivudine $(300$ $\mathrm{mg})$ or dolutegravir $(50 \mathrm{mg})$ plus tenofovir disoproxil fumarate $(300 \mathrm{mg}) /$ emtricitabine $(200 \mathrm{mg})$.

The primary endpoint of the GEMINI studies was proportion of participants with plasma VL $<50$ copies $/ \mathrm{mL}$ at week 48 according to the Snapshot algorithm in the intentionto-treat-exposed (ITT-E) population. VL decline to $<50$ copies/mL was evaluated as median change in $\log _{10}$-transformed VL from baseline at weeks 4, 8, 12, 16, 24, 36, and 48 , and time to viral suppression (plasma VL $<50$ copies $/ \mathrm{mL}$ ) was determined using the nonparametric Kaplan-Meier method. Proportion of participants with plasma VL $<50$ copies/mL at weeks $4,8,12,16,24,36$, and 48 according to the Snapshot algorithm were compared between the 2DR and 3DR groups by baseline VL. The Abbot RealTime HIV-1 assay used to measure VLs has a lower limit of detection of 40 copies $/ \mathrm{mL}$. Therefore, we conducted an exploratory analysis where VLs from 40 to $10,000,000$ copies/mL were reported qualitatively as target detected or target not detected (TND) for samples with VL $<40$ copies/mL. In addition, TND data were used to compare the proportion of participants with undetectable VL summarized by visit and at week 48 by baseline VL.

$\mathrm{CD}^{+}$cell count by baseline VL $(>100,000$ and $\leq 100,000$ copies $/ \mathrm{mL}$ ) was determined at week 48 , and change from baseline was estimated using an ANCOVA model adjusting for study (GEMINI-1 vs GEMINI-2), baseline plasma VL, baseline $\mathrm{CD}^{+}$cell count, subgroup, and treatment by relevant subgroup interaction.

\section{RESULTS}

\section{Participant Characteristics}

As previously reported, baseline demographics were similar between the 2DR and 3DR groups in the pooled GEMINI-1 and GEMINI-2 ITT-E populations. ${ }^{3}$ Median age (range) was $32(18-72)$ years in the 2DR group and 33 $(18-70)$ years in the 3DR group, and 16\% (113/716) and 14\% (98/717) of participants in the 2DR and 3DR groups, respectively, were women. Most participants were white [2DR, 67\% (480/716); 3DR, 69\% (497/717)] and nonHispanic/Latino [2DR, 70\% (501/716); 3DR, 68\% (485/ 717)].

Both groups were well-balanced regarding proportion of participants in each $\mathrm{VL}$ and $\mathrm{CD}^{+}$cell count strata. A majority of participants in each group had VL $\leq 100,000$ copies $/ \mathrm{mL}$ at baseline [2DR, 80\% $(\mathrm{n}=576) ; 3 \mathrm{DR}, 79 \%(\mathrm{n}=$ 564)]. Twenty percent $(n=140)$ of participants in the $2 \mathrm{DR}$ group and $21 \%(\mathrm{n}=153)$ in the 3DR group had baseline VL $>100,000$ copies $/ \mathrm{mL} ; 7 \%(\mathrm{n}=51)$ and $6 \%(\mathrm{n}=46)$ had $>250,000$ copies $/ \mathrm{mL} ; 3 \%(\mathrm{n}=18)$ and $3 \%(\mathrm{n}=24)$ had 
$>400,000$ copies $/ \mathrm{mL}$; and $2 \%(\mathrm{n}=13)$ and $2 \%(\mathrm{n}=15)$ had $>500,000$ copies $/ \mathrm{mL}$ (after screening VL $\leq 500,000$ copies $/ \mathrm{mL}$ ). Median (range) $\mathrm{CD}^{+}$cell count was 427 (19-1399) cells $/ \mathrm{mm}^{3}$ in the 2DR group and $438(19-1497)$ cells $/ \mathrm{mm}^{3}$ in the 3DR group. In total, $9 \%(63 / 716)$ and $8 \%$ (55/717) of participants in the 2DR and 3DR groups, respectively, had $\leq 200$ cells $/ \mathrm{mm}^{3}$.

\section{Virologic Response by Baseline VL}

Median change in VL from baseline at week 4 was -2.77 and $-2.80 \log _{10}$ copies $/ \mathrm{mL}$ in the overall population of the 2DR and 3DR groups, respectively. ${ }^{3}$ In participants with baseline VL $>100,000$ copies $/ \mathrm{mL}$, median change from baseline at week 4 was -3.38 and $-3.40 \log _{10}$ copies $/ \mathrm{mL}$ in the 2DR and 3DR groups, respectively. In both the overall population and in those with baseline VL $>100,000$ copies $/ \mathrm{mL}$, reduction in VL was maintained through 48 weeks. Viral suppression (VL $<50$ copies $/ \mathrm{mL}$ ) was achieved at a median of 29 days (week 4 visit) in both groups in the overall study population [hazard ratio (HR), 1.01; 95\% confidence interval (CI): 0.91 to 1.12 ] and in those with baseline VL $\leq 100,000$ copies/mL (HR, 0.99 ; $95 \%$ CI: 0.88 to 1.11); for participants with baseline $\mathrm{VL}>100,000$ copies $/ \mathrm{mL}$, viral suppression was achieved at a median of 57 days (week 8 visit) in both groups (HR, 1.00; 95\% CI: 0.79 to 1.26 ).

Overall, $72 \%(518 / 716)$ of participants in the 2DR group and $70 \%(504 / 717)$ in the 3DR group achieved viral suppression (VL $<50$ copies $/ \mathrm{mL}$ ) at week 4 . For participants with baseline VL $\leq 100,000$ copies $/ \mathrm{mL}$, based on the Snapshot algorithm, 83\% (477/576) in the 2DR group and $81 \%(456 / 564)$ in the 3DR group achieved viral suppression by week 4 compared with $29 \%(41 / 140)$ and $31 \%(48 / 153)$ of participants with baseline VL $>100,000$ copies $/ \mathrm{mL}$ in the 2DR and 3DR groups, respectively (Fig. 1A). At week 24, the proportion of participants with VL $<50$ copies $/ \mathrm{mL}$ was similarly high in both groups, regardless of baseline VL, and the response was sustained through week 48. Across all baseline VL strata, high and similar rates of viral suppression were observed in the Snapshot analysis at week 48 in both groups, including in participants whose VL increased to exceed 500,000 copies $/ \mathrm{mL}$ between the screening and baseline assessments (Fig. 1B).

Assessment of qualitative TND data showed 34\% (246/ $716)$ in the $2 \mathrm{DR}$ group and $32 \%(228 / 717)$ in the $3 \mathrm{DR}$ group had undetectable VL at week 4, and $77 \%(553 / 716)$ in the 2DR group and $73 \%(525 / 717)$ in the 3DR group had undetectable VL at week 48 (Fig. 2A). Among participants with baseline VL $>100,000$ copies $/ \mathrm{mL}, 64 \%(90 / 140)$ in the 2DR group and $52 \%(79 / 153)$ in the 3DR group had undetectable VL at week 48 (unadjusted treatment difference, $12.7 \%$; 95\% CI: 1.4 to 23.9 ; Fig. 2B). Proportions of participants with undetectable VL were similar between treatment groups in the other baseline VL subgroups.

\section{Change in $\mathrm{CD}^{+}$Cell Count by Baseline VL}

For participants with baseline VL $\leq 100,000$ copies $/ \mathrm{mL}$, median (interquartile range) $\mathrm{CD}^{+}$cell count increased by 206
(109-303) cells $/ \mathrm{mm}^{3}$ in the 2DR group and by 191 (101-304) cells $/ \mathrm{mm}^{3}$ in the 3DR group at week 48 [adjusted mean difference $(95 \% \mathrm{CI}), 7.2(-14.6$ to 29.0$)$ cells $\left./ \mathrm{mm}^{3}\right]$. For participants with baseline VL $>100,000$ copies $/ \mathrm{mL}$, median (interquartile range) $\mathrm{CD}^{+}$cell count increased by 233 (151-344) cells $/ \mathrm{mm}^{3}$ in the 2DR group and by 211 (125-359) cells $/ \mathrm{mm}^{3}$ in the 3DR group at week 48 [adjusted mean difference $(95 \% \mathrm{CI}), 7.4$ (-35.9 to 50.8$)$ cells $\left./ \mathrm{mm}^{3}\right]$.

\section{DISCUSSION}

In the GEMINI studies, the 2DR dolutegravir plus lamivudine was associated with a rapid rate of decline in VL, comparable to that achieved with the 3DR dolutegravir plus tenofovir disoproxil fumarate/emtricitabine, with most participants achieving virologic suppression within 4 weeks at the $<50$ copies $/ \mathrm{mL}$ level. In addition, proportion of participants with undetectable VL and TND using the Abbott RealTime assay was similar between groups over time through week 48 and also across VL strata at week 48. These data demonstrate the potency of dolutegravir plus lamivudine in the treatment of HIV-1 infection, similar to that of a standard-of-care 3DR.

An update to the US Department of Health and Human Services (DHHS) treatment guidelines based on the GEMINI results supports the use of dolutegravir plus lamivudine as initial therapy for HIV-1 infection in PLHIV who have HIV-1 RNA $\leq 500,000$ copies $/ \mathrm{mL}$, no active hepatitis B infection, and available HIV genotype information. ${ }^{6}$ According to the DHHS, demonstration of long-term efficacy of the 2DR dolutegravir plus lamivudine supports the recommendation of this regimen for use as initial therapy. A preplanned analysis of the GEMINI studies showed that dolutegravir plus lamivudine maintained noninferior efficacy compared with dolutegravir plus tenofovir disoproxil fumarate/emtricitabine at 96 weeks, with low rates of confirmed virologic withdrawal and no treatment-emergent INSTI or NRTI mutations. ${ }^{7}$

A question regarding the adoption of 2DRs in favor of the mainstay $3 \mathrm{DRs}$ is whether 2DRs can provide equivalent efficacy. ${ }^{2}$ Treatment guidelines have considered VL $>100,000$ copies $/ \mathrm{mL}$ and $\mathrm{CD}^{+}$cell count $<200$ cells $/ \mathrm{mm}^{3}$ as limitations for the use of $2 \mathrm{DRs},{ }^{4}$ and a shift toward widespread use of 2DRs will require demonstration that the regimen is sufficiently potent to provide virologic suppression in these populations. In a single-arm study of raltegravir plus ritonavir-boosted darunavir, baseline VL $>100,000$ copies $/ \mathrm{mL}$ (HR, $3.76 ; 95 \%$ CI: 1.52 to $9.31 ; P=0.004$ ) and lower $\mathrm{CD}^{+}$cell count (HR, 0.77 ; $95 \%$ CI: 0.61 to 0.98 cells per 100 cells $/ \mathrm{mm}^{3}$ increase; $P=0.037$ ) were associated with virologic failure, ${ }^{8}$ and in a randomized trial, raltegravir plus ritonavir-boosted darunavir was noninferior to a $3 \mathrm{DR}$ of raltegravir plus 2 NRTIs in the overall study population, but inferior in participants with baseline VL $>100,000$ copies $/ \mathrm{mL}$ and $\mathrm{CD}^{+}$cell count $<200$ cells $/ \mathrm{mm}^{3}{ }^{5}{ }^{5}$ These trials included participants with baseline VL $>500,000$ copies $/ \mathrm{mL}$, although patient numbers in these subgroups were small, as in the GEMINI studies. The data described here demonstrate that dolutegravir plus lamivudine has similar efficacy to dolutegravir plus tenofovir disoproxil fumarate/emtricitabine in participants with baseline VL $>100,000$ copies $/ \mathrm{mL}$, with no 


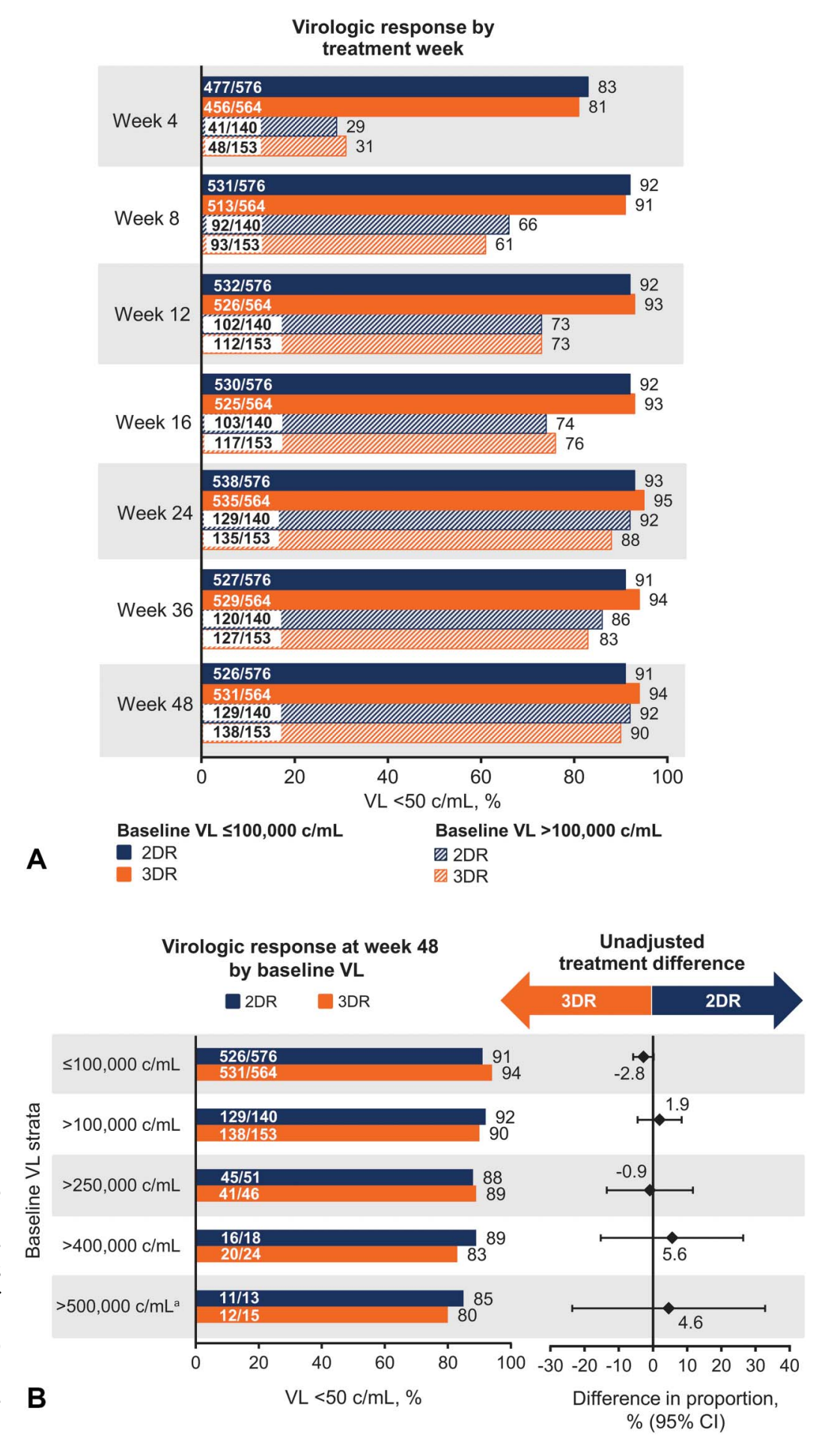

$$
\begin{array}{lll}
\text { Baseline } \mathrm{VL} \leq 100,000 \mathrm{c} / \mathrm{mL} & \text { Baseline } \mathrm{VL}>100,000 \mathrm{c} / \mathrm{mL} \\
2 \mathrm{DR} & & 2 \mathrm{DR} \\
3 \mathrm{DR} & & 3 \mathrm{DR}
\end{array}
$$

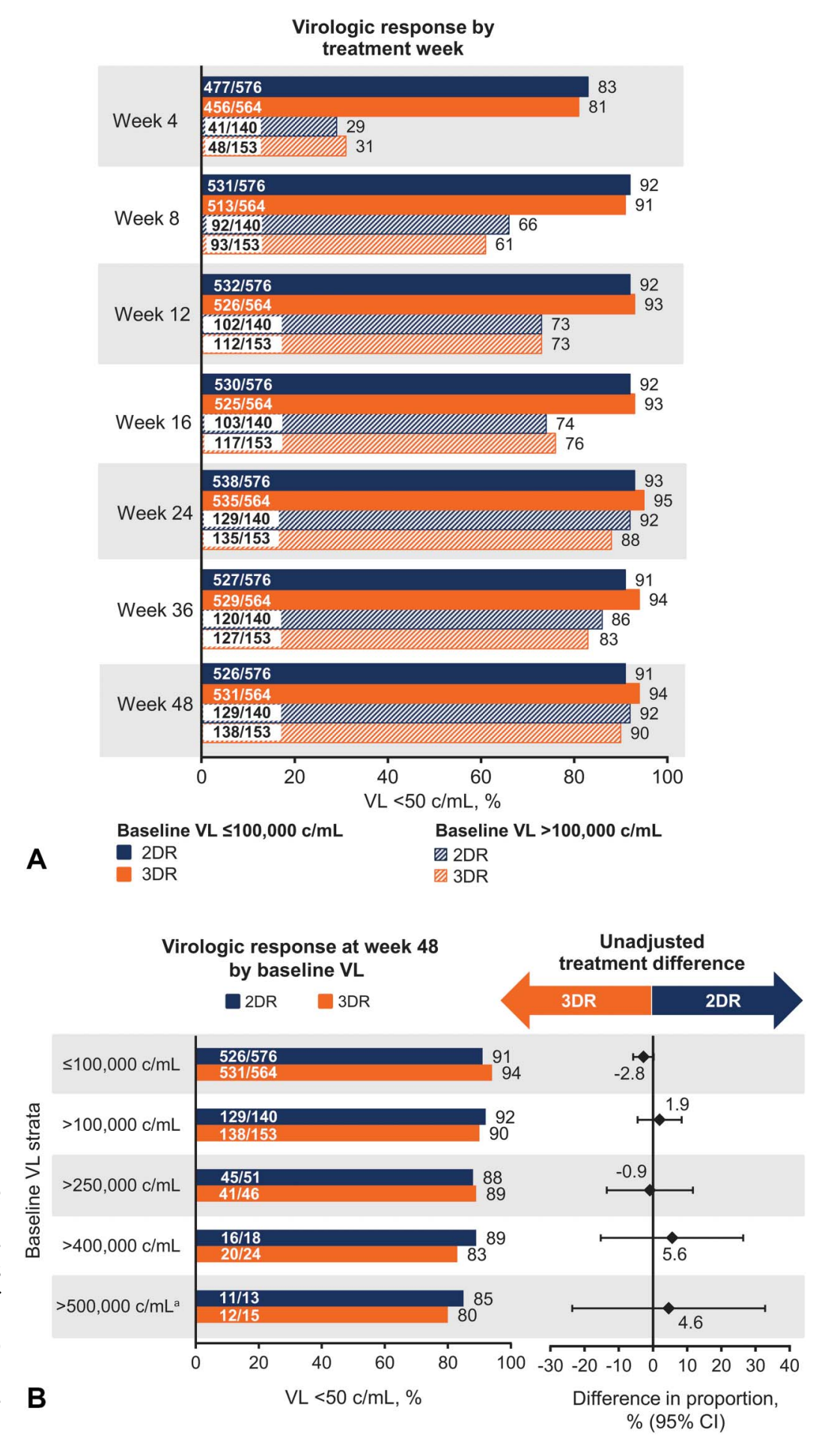

FIGURE 1. Snapshot analysis of virologic response $(\mathrm{VL}<50$ copies $/ \mathrm{mL}$ ) (A) by treatment week and (B) at week 48 by baseline $V L$, in the ITT-E population. 2DR, 2-drug regimen (dolutegravir plus lamivudine); 3DR, 3-drug regimen (dolutegravir plus tenofovir disoproxil fumarate/emtricitabine). aparticipants were required to have $\mathrm{VL} \leq 500,000$ copies/mL at screening; however, VL had increased to exceed 500,000 copies $/ \mathrm{mL}$ at baseline in some participants.

indication of declining activity in the higher VL strata. Not unexpectedly, the time to achieve virologic suppression was longer (and similar) in both groups for participants with baseline VL $>100,000$ copies/mL, whereas both baseline VL subgroups in both treatment groups were suppressed at the week 24 time point. This difference across populations is difficult to apply to a clinical decision regarding the concept of $\mathrm{U}=\mathrm{U}$ (undetectable virus = untransmittable virus), which should be based on individual VL results and clinical circumstances.

Previously it was shown that for the small subset of participants with baseline $\mathrm{CD} 4^{+}$cell count $\leq 200$ cells $/ \mathrm{mm}^{3}$, a lower proportion in the $2 \mathrm{DR}$ group achieved virologic suppression compared with the 3DR group [79\% (50/63) vs 


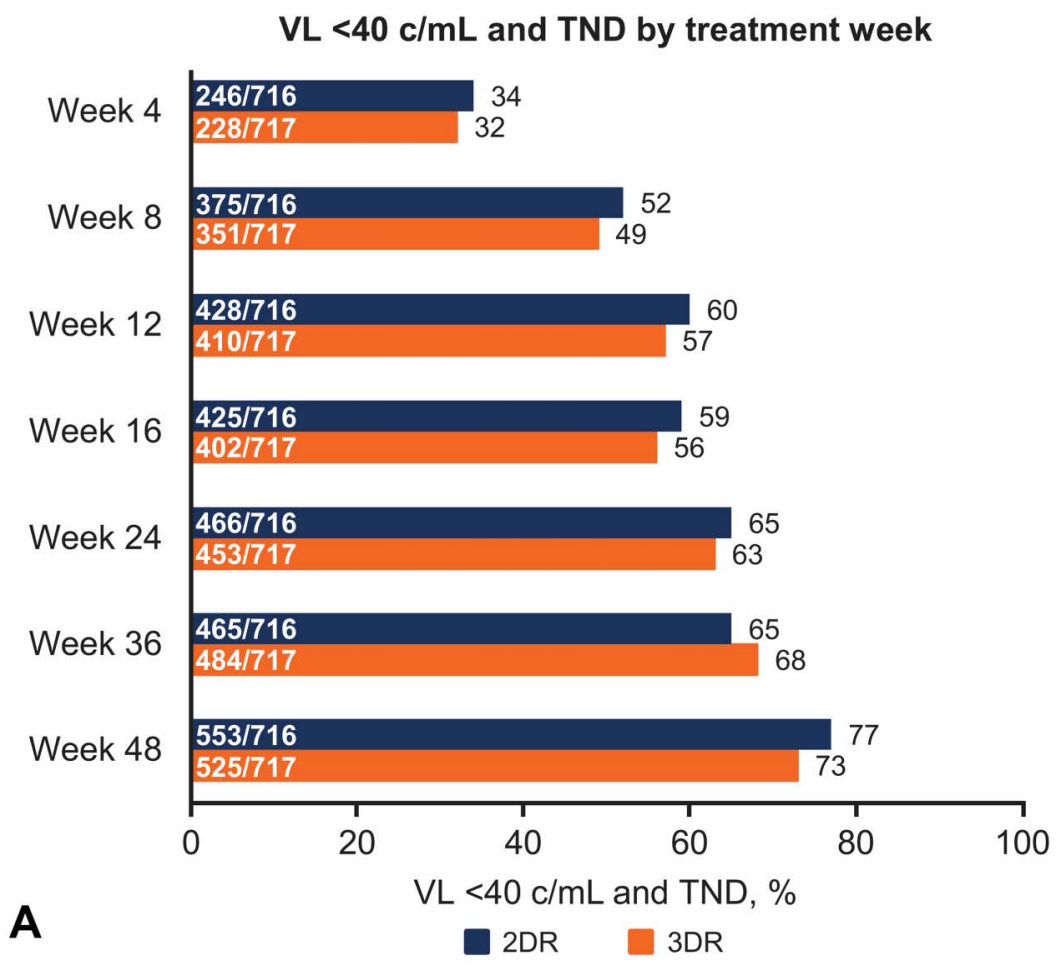

FIGURE 2. Proportion of participants with $\mathrm{VL}<40$ copies/mL and TND (as assessed by Abbott RealTime HIV-1 assay) (A) by treatment week and (B) at week 48 by baseline VL, in the ITT-E population. 2DR, 2-drug regimen (dolutegravir plus lamivudine); 3DR, 3-drug regimen (dolutegravir plus tenofovir disoproxil fumarate/emtricitabine).

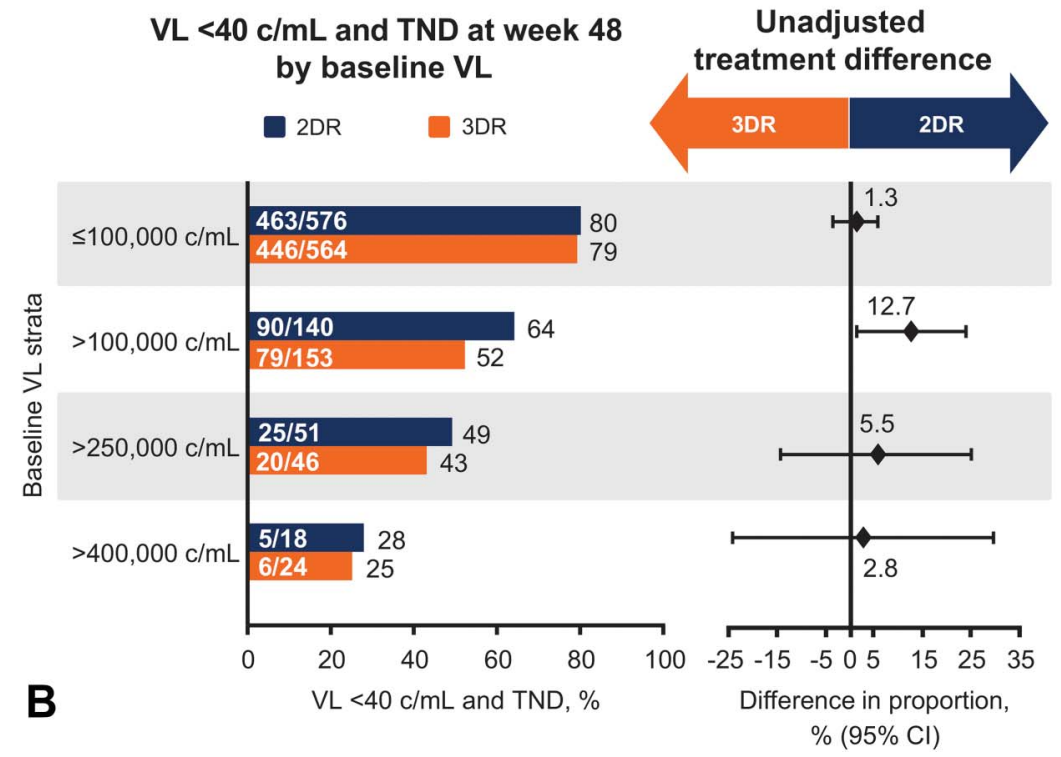

$93 \%(51 / 55)]^{3}$ The reasons for failure for most of the participants in this subgroup were unrelated to virologic efficacy or treatment-related adverse events. In this subgroup, only $5 \%(3 / 63)$ in the $2 \mathrm{DR}$ group and $2 \%(1 / 55)$ in the $3 \mathrm{DR}$ group had $\mathrm{VL} \geq 50$ copies $/ \mathrm{mL}$ at week 48 . Only 1 participant in the 2DR group and none in the $3 \mathrm{DR}$ group met confirmed virologic withdrawal criteria.

The GEMINI studies had several limitations, as described elsewhere. ${ }^{3}$ The study populations were predomi- nantly composed of men under age 50 and are not fully reflective of the global population of PLHIV. Furthermore, the exclusion of participants with $\mathrm{VL}>500,000$ copies $/ \mathrm{mL}$ at screening and the small numbers of participants with VL $>500,000$ copies $/ \mathrm{mL}$ at baseline preclude consideration of the use of dolutegravir plus lamivudine in those with very high VLs.

This post-hoc analysis of the GEMINI studies demonstrates that a 2DR of dolutegravir plus lamivudine that 
reduces cumulative drug exposure was as potent as a standard 3DR in suppressing viral replication in PLHIV with VL up to 500,000 copies $/ \mathrm{mL}$.

\section{REFERENCES}

1. Marcus JL, Chao CR, Leyden WA, et al. Narrowing the gap in life expectancy between HIV-infected and HIV-uninfected individuals with access to care. J Acquir Immune Defic Syndr. 2016;73:39-46.

2. Achhra AC, Mwasakifwa G, Amin J, et al. Efficacy and safety of contemporary dual-drug antiretroviral regimens as first-line treatment or as a simplification strategy: a systematic review and meta-analysis. Lancet $H I V .2016 ; 3: \mathrm{e} 351-\mathrm{e} 360$.

3. Cahn P, Madero JS, Arribas JR, et al. Dolutegravir plus lamivudine versus dolutegravir plus tenofovir disoproxil fumarate and emtricitabine in antiretroviral-naive adults with HIV-1 infection (GEMINI-1 and GEMINI-2): week 48 results from two multicentre, double-blind, randomised, non-inferiority, phase 3 trials. Lancet. 2019;393:143-155.
4. Kelly SG, Nyaku AN, Taiwo BO. Two-drug treatment approaches in HIV finally getting somewhere? Drugs. 2016;76:523-531.

5. Raffi F, Babiker AG, Richert L, et al. Ritonavir-boosted darunavir combined with raltegravir or tenofovir-emtricitabine in antiretroviralnaive adults infected with HIV-1: 96 week results from the NEAT001/ ANRS143 randomised non-inferiority trial. Lancet. 2014;384:1942-1951.

6. Panel on Antiretroviral Guidelines for Adults and Adolescents. Guidelines for the Use of Antiretroviral Agents in Adults and Adolescents Living with $H I V$. Department of Health and Human Services; 2019. Available at: https://aidsinfo.nih.gov/contentfiles/lvguidelines/adultandadolescentgl. pdf. Accessed February 4, 2020.

7. Cahn P, Madero JS, Arribas JR, et al. Durable efficacy of dolutegravir plus lamivudine in antiretroviral treatment-naive adults with HIV-1 infection 96-week results from the GEMINI-1 and GEMINI-2 randomized clinical trials. J Acquir Immune Defic Syndr. 2019 [Epub ahead of print].

8. Taiwo B, Zheng L, Gallien S, et al. Efficacy of a nucleoside-sparing regimen of darunavir/ritonavir plus raltegravir in treatment-naive HIV-1infected patients (ACTG A5262). AIDS. 2011;25:2113-2122. 